
allemande

47-1| 2015

L'Allemagne et l'Europe | La déprise de l'Empire napoléonien en Allemagne en 1813

Des alliés aux ennemis : le rôle des forces de la Tierce Allemagne dans les occupations du territoire français (1814 et 1815-1818)

\title{
Christine Haynes
}

\section{(2) OpenEdition}

\section{Journals}

Édition électronique

URL : https://journals.openedition.org/allemagne/472

DOI : 10.4000/allemagne.472

ISSN : 2605-7913

Éditeur

Société d'études allemandes

Édition imprimée

Date de publication : 26 juin 2015

Pagination : 163-175

ISSN : 0035-0974

Référence électronique

Christine Haynes, «Des alliés aux ennemis : le rôle des forces de la Tierce Allemagne dans les occupations du territoire français (1814 et 1815-1818) », Revue d'Allemagne et des pays de langue allemande [En ligne], 47-1 | 2015, mis en ligne le 13 décembre 2017, consulté le 19 mai 2021. URL http://journals.openedition.org/allemagne/472 ; DOI : https://doi.org/10.4000/allemagne.472 


\section{Des alliés aux ennemis: le rôle des forces de la Tierce Allemagne dans les occupations du territoire français (1814 et 1815-1818)}

\section{- Christine Haynes*}

« [Am Lauerheim an der Lauer] Ein Teil [der Preußische Wache] kam zu uns ins Quartier und hatte Rasttag. Diese Leute waren kaum zwei Stunden im Quartier, so wurden schon die Einwohner von ihnen mißhandelt und die Gardisten betranken sich größtenteil mit Schnaps. Das Dorf war preußisch... In dieser Zeit [4. Juli] wurde die Schlacht bei Waterloo in den Niederlanden zwischen PREUSSEN \& ENGLAND einerseits \& den Französen unter Napoleon anderseits geschlagen, wobei die französische Armee vollständig geschlage \& auseinander gesprengt wurde. Es rückten nun sofort alle Armeen in Frankreich ein ${ }^{(1)}$.

Il est bien connu que l'armée prussienne figura, pendant les deux crises et chutes de l'Empire de 1813-1814 et de 1815, parmi les ennemis les plus farouches de la France napoléonienne. De la défense de Berlin et du champ de bataille de Leipzig, en 1813, jusqu'à la campagne de France de 1814 et la plaine de Waterloo en 1815, c'est une guerre d'annihilation que livra l'état-major prussien contre Napoléon, porté qu'il était par l'esprit de revanche sur la défaite subie en 1806 à Iéna. Cette défaite avait valu à la Prusse de connaître l'humiliation d'une lourde amputation territoriale, de l'entretien d'une armée d'occupation et du règlement d'une indemnité de guerre de $140 \mathrm{M}$ de francs, réduite plus tard à $120 \mathrm{M}$, tout en étant forcée de se joindre à l'alliance contre l'Angleterre et au blocus continental. Le souvenir de ce traumatisme devait conditionner le comportement des officiers et des soldats prussiens lors des campagnes de 1814 et 1815 contre leur pire ennemi. En 1815 en particulier, l'esprit de vengeance de l'armée prussienne dirigée par le maréchal Blücher ne fut contenu que grâce à l'intervention des Anglais. En plus de ses manœuvres destinées à détruire le pont d'Iéna sur la Seine,

* Professeur associée d'histoire à l'University of North Carolina, Charlotte (États-Unis).

1 Journal du lieutenant Johann Michael Anstlperger, du $2^{\mathrm{e}}$ régiment d'infanterie de Bavière "Kronprinz » (1797-1818), au moment de la bataille de Waterloo (Abschrift der Kriegstagebücher in der Hdschr. Slg. III Kriegs- \& Heeresgeschichte im Allgemeinen Nr. 56, Bäyerisches Hauptstaatsarchiv Kriegsarchiv, HS 442/2). 
qui échouèrent grâce au duc de Wellington, Blücher est connu pour avoir imposé une lourde contribution financière à la ville de Paris et organisé la récupération des œuvres d'art spoliées par Napoléon. Le maréchal prussien a aussi autorisé - en réalité, même, encouragé - ses troupes à perpétrer de nombreuses atrocités dans la capitale française et ailleurs. À qui s'en plaignait, Blücher rappelait les brutalités commises par Daru en Prusse en 1806, et répondait, sarcastique: "Quoi? Elles n'ont fait que cela? Elles auraient pu faire bien davantage " ${ }^{(2)}$. Même après qu'il fut rentré en Prusse, au terme de la première occupation militaire de 1815 , les soldats prussiens demeurèrent les plus redoutés parmi tous les contingents alliés qui poursuivirent l'occupation de garantie dans sept départements de la frontière nord-est de la fin 1815 à la fin 1818. Longtemps après, les récits de leurs atrocités restèrent vivants dans la tradition orale de ces régions, où l'on donnait aux chiens enragés le sobriquet de «Blücher », spécialement pendant les autres épisodes d'invasion qu'elles subirent, ainsi en 1870-1871. Cette mémoire collective, en retour, se répercuta sur l'historiographie de la Prusse, non seulement en France mais en Allemagne même où l'on voit la fin des guerres napoléoniennes comme des "guerres de libération », et où l'on recycla les mêmes récits pour illustrer l'atmosphère de vengeance et de brutalité qui entoura les forces prussiennes, dans l'invasion alliée comme d'ailleurs dans l'occupation du territoire français ${ }^{(3)}$.

Dans une moindre mesure, pour l'historiographie savante comme pour la tradition populaire, les Autrichiens, ainsi que les "Cosaques ", sont enregistrés comme des occupants cruels, en 1814 comme en 1815-1818, de nouveau du fait qu'ils avaient une revanche à prendre sur la France qui avait occupé leur propre pays (pour les premiers en 1805 et 1809, et pour les seconds en 1812). En 1815 par exemple, les Autrichiens se distinguèrent en menaçant d'annexer des portions du territoire français à l'est, en s'immisçant dans les affaires des autorités locales, et en réquisitionnant le produit des impôts ${ }^{(4)}$.

Beaucoup moins étudiés mais également importants pour l'histoire de ces occupations sont les pays de la Tierce Allemagne, ces petits États souverains souvent alliés à

2 Cité par Emmanuel de Waresquiel et Benoît Yvert, Histoire de la Restauration, 1814-1830: Naissance de la France moderne, Paris, Librairie Académique Perrin, 1996, p. 147.

3 Sur l'attitude des troupes prussiennes en 1814-1815, voir également Marc BLANCPAIN, La vie quotidienne dans la France du Nord sous les occupations, 1814-1944, Paris, Hachette, 1983, notamment p. 57 ; Jacques Hantraye, Les Cosaques aux Champs-Élysées: L'occupation de la France après la chute de Napoléon, Paris, Belin, 2005; et les travaux de Michael V. Leggiere, Napoleon/Berlin: The Franco-Prussian War in North Germany, 1813, Norman, University of Oklahoma Press, 2002, qui écrit p. 295: « La haine de Blücher vis-à-vis de Napoléon, ajoutée au désir de Gneisenau de répondre aux visites des villes allemandes par les Français par des incursions en retour, aboutit à ce que des milliers de soldats allemands et russes perdirent la vie en 1814 après que l'Allemagne eut été libérée. » Sur l'héritage de la défaite de 1806, voir aussi Karen Hagemann, " Mannlicher Muth und Teutsche Ehre »: Nation, Militär und Geschlecht zur Zeit der antinapoleonischen Kriege Preußens, Paderborn, Ferdinand Schöning, 2002; Volker WACKER, Die allierte Besetzung Frankreichs in den Jahren 1814 bis 1818, Hambourg, Verlag Dr. Kovač, 2001; et Karl Овеrmann, " La situation de la Prusse sous l'occupation française entre 1807 et 1813 », in: G. JaCQUemyns (dir.), Occupants/ Occupés, 1792-1815: Colloque de Bruxelles, 29 et 30 janvier 1968, Bruxelles, Université Libre de Bruxelles / Éditions de l'Institut de Sociologie, 1969, p. 257-286.

4 Pour les Autrichiens, voir Robert Ouvrard, 1809: Les Français à Vienne: Chronique d'une occupation, Paris, Nouveau Monde Éditions / Fondation Napoléon, 2009, ainsi que Paul Gaffarel, $L a$ Seconde Restauration et la Seconde Occupation autrichienne à Dijon (juin-déc. 1815), Dijon, Imprimerie Darantière, s.d. 
la France à l'issue de la dissolution du Saint-Empire entre 1806 et 1813 . Remodelée par des forces exogènes, cette région dut lutter tout au long de cette période pour trouver sa voie entre la Prusse et l'Autriche aussi bien qu'entre Napoléon et ses adversaires coalisés. Ainsi que l'a noté James Sheehan, entre l'occupation de la Rhénanie par les Français en 1794 et la bataille de Leipzig en 1813, quelque $60 \%$ de la population allemande changèrent de souverain sous la pression des troupes françaises ${ }^{(5)}$. Cette Tierce Allemagne joua en France un rôle important, et souvent dévastateur, en 1814 et en 1815-1818. Alors que ses contingents militaires sont souvent oubliés dans l'histoire de ces invasions et occupations de la France, les sources montrent que les forces issues de l'ancienne Confédération du Rhin n'étaient pas moins brutales à l'égard des vaincus, et parfois l'étaient plus, que leurs homologues prussiennes et autrichiennes. Ralliées tardivement à la coalition, poussées soit par une animosité envers les Français, soit par une volonté de démontrer leur allégeance au camp anti-napoléonien, soit les deux à la fois, elles firent preuve d'une exceptionnelle hostilité envers les populations occupées. On illustrera cet argument avec trois cas, la Saxe, la Bavière et le Wurtemberg. Après un bref rappel de leur parcours pendant l'ère napoléonienne, on s'appuiera sur la documentation relative aux régions où les troupes de ces pays ont été stationnées ou bien qu'elles ont traversées, ainsi que sur les archives militaires allemandes proprement dites ${ }^{(6)}$.

\section{La déprise de l'Empire napoléonien : deux occupations successives}

À la suite de la bataille de Leipzig, les Alliés poursuivirent leur campagne contre Napoléon et pénétrèrent en France le jour de l'an 1814. Fin mars, ils avaient marché jusqu'à Paris, qu'ils occupèrent tout le printemps; mais avec l'abdication et l'exil de Napoléon, ils rentrèrent de manière à laisser la reconstruction du pays à la monarchie bourbonienne restaurée. Par contraste avec l'occupation de 1814, relativement courte et plutôt bienveillante, celle de 1815 fut longue et pénible. Après Waterloo, la France fut envahie par plus d'un million de soldats, qui se répartirent sur la plus grande partie du territoire et restèrent au moins jusqu'à la fin de l'année. Le second traité de Paris du 20 novembre 1815 en laissait 150000 pour une " occupation de garantie ", jusqu'à ce que les Alliés fussent indemnisés, à hauteur de $700 \mathrm{M}$ de francs, des préjudices causés par le retour de Napoléon (sans compter ni les frais de ravitaillement, d'équipement et de soldes, qui ensemble se montaient à $150 \mathrm{M}$ de francs par an, ni les réclamations d'indemnisations des individus étrangers). Projetée au départ pour cinq ans et terminée finalement au bout de trois, l'occupation engagea plus de 30000 hommes de chacune des quatre grandes puissances (Angleterre, Russie, Prusse, Autriche) et

5 James Sheenan, German History, 1770-1866, Oxford, Clarendon Press, 1989, p. 251.

6 Les sources allemandes de cet article sont surtout les archives militaires relatives au « Feldkrieg gegen Frankenreich " de 1814, et au "Besetzung " ou " Okkupationskorps » en France entre 1815 et 1818. Pour la Saxe: Hauptstaatsarchiv Dresden, surtout Bestände 10025 (Geheimes Konsilium), 10026 (Geheimes Kabinett), et 11339 (Generalstab: Generaloberkommando); pour la Bavière: Bäyerisches Hauptstaatsarchiv Kriegsarchiv, Munich, Alter Bestand B, liasse 587-607, "Krieg gegen Frankreich ", ainsi que la riche collection de journaux manuscrits et de journaux de route tenus par des officiers et soldats bavarois; pour le Wurtemberg: Hauptstaatsarchiv Stuttgart, surtout Bestand E 270 a, liasse 463-473, Geheime Kriegskanzlerei: Occupationskorps im Elsaß, et Bestand E 289 a, liasse 275284: Bezeichnung des Bestands: Mobile Kommandobehörden I. 
30000 issus de plus petits États (Danemark, Hanovre, Saxe, Hesse, Bade, Bavière, Wurtemberg), qui furent stationnés dans 18 villes de garnison réparties le long de la frontière nord-est, depuis la Manche jusqu’à la Suisse.

Deux points sont à rappeler concernant la Tierce Allemagne. D'abord, que les armées de 1814-1815 étaient multinationales. Ainsi pendant l'invasion de 1815 les Hanovriens étaient-ils mélangés avec des soldats anglais, danois et hollandais, tandis que les Wurtembergeois et les Badois côtoyaient des Autrichiens. Pour l'occupation de garantie, néanmoins, on assigna à chaque pays une zone particulière le long de la frontière nord-est. Les Prussiens, commandés par le comte von Zieten, furent stationnés à Sedan; les Autrichiens, sous les ordres du baron von Frimont, à Colmar; les Saxons du major général Gablentz étaient à Tourcoing; les troupes du Wurtemberg, dont le chef était le lieutenant général baron von Woellworth (qui céda la place à Scheler en novembre 1816), étaient à Wissembourg; et enfin les Bavarois, commandés par le lieutenant général de Lamotte, étaient cantonnés aux alentours de Sarreguemines. Cela rendait plus facile aux Français de 1815 de distinguer les troupes d'occupation en fonction du critère de nationalité - et il en va de même aujourd'hui pour l'historien. On peut même soutenir que cette mission d'occupation eut pour effet de promouvoir une identité sub-nationale au sein de ces corps d'armée. Deuxièmement, il faut noter que la présence de ces troupes étrangères sur le sol français s'effectuait dans un contexte de grande instabilité. Dans la "vacance du pouvoir " provoquée par l'effondrement de l'Empire, il restait de grandes incertitudes concernant l'avenir de la Tierce Allemagne: dans quelle mesure ces États se fondraient-ils dans un ensemble plus grand, sous influence prussienne ou autrichienne? Quelles seraient leurs frontières? Leur constitution? Ces interrogations exacerbaient les tensions entre Prusse et Autriche aussi bien qu'entre les États de la Confédération, des tensions très anciennes qui remontaient au-delà des guerres napoléoniennes où par ailleurs certains avaient été ennemis (Saxe et Prusse par exemple) ou occupants (le Wurtemberg en Autriche) les unes pour les autres. Ce ne fut pas sans influence sur le comportement des troupes de la Tierce Allemagne en France.

\section{La Saxe}

C’était particulièrement vrai pour la Saxe, qui avait été, prise en tenaille entre Prusse et Autriche, une alliée solide de la France depuis la guerre de Sept Ans. Bien que partie du côté prussien au moment de l'invasion de 1806, la Saxe avait vite changé de bord et était rentrée dans la Confédération du Rhin. Sous Frédéric Auguste, qui y gagna un titre royal, elle demeura fidèle à Napoléon jusqu'à août 1813, combattant avec les Français face aux Prussiens dans la campagne de Berlin. Mais après que la campagne se fut orientée vers Dresde, et eut dévasté les environs de la ville, l'alliance suscita de plus en plus de frustrations, au point que les troupes changèrent de camp à la bataille de Leipzig. Trop tard sans doute pour éviter que le roi fût arrêté pour trahison par les Prussiens ${ }^{(7)}$, et pour éviter de grosses pertes au congrès de Vienne $\left(20000 \mathrm{~km}^{2}\right.$ et 850000 habitants), donc pour affaiblir fortement la position du pays. Mais pendant l'invasion de 1814, la Saxe mit sur le terrain 20000 hommes issus de la milice, et 20000

7 M. Leggiere, Napoleon/ Berlin (note 3), p. 220. 
des troupes de ligne, commandés par le baron Friedrich von Langenau. Lannée suivante, malgré des mutineries intervenues en mai, elle parvint à mettre 16000 hommes à la disposition des Alliés (sans compter les contributions de la Saxe-Cobourg, de la Saxe-Gotha, de la Saxe-Weimar ${ }^{(8)}$... À partir de novembre 1815, 5000 Saxons demeurèrent sur le sol français, autour de Tourcoing (dont 686 hommes et 300 chevaux dans la ville proprement dite) ${ }^{(9)}$.

Les sources font apparaître le corps d'occupation saxon comme plutôt modéré, moins préoccupé de châtier les Français que de consolider la position du pays vis-à-vis des Alliés, surtout de la Prusse, et de préserver le Trésor royal. Néanmoins, si l’on se fie aux documents des Archives municipales de Tourcoing, les troupes furent exigeantes, provocatrices et souvent brutales vis-à-vis des Français. Le général von Gablenz, comme ses homologues commandant les forces des autres puissances, réclama pour ses appartements une longue liste de meubles et d'objets (draps et serviettes pour luimême et ses domestiques, nappes et linge de table, services complets avec double série d'assiettes, toute sorte de pots et casseroles: il y en avait pour 5000 francs $\left.^{(10)}\right)$. Son second, le lieutenant Bucher, qui commandait à Tourcoing, multipliait les demandes erratiques: la réparation du lit et le remplacement du miroir pour la chambre de l'officier commandant sa garde (juillet 1816), un nouveau fourreau pour le couteau de son fournisseur en boucherie (août 1817), la réparation d'un plafond en pierre dans une courette pour éviter que les eaux ne s'écoulent dans la chambre d'un officier des gardes et n'y répandent « des odeurs infectées » (octobre 1816), l'agrandissement d'un croisillon afin de faire pénétrer plus de lumière dans l'écurie où étaient stationnés les chevaux des Saxons, dans une auberge des alentours (novembre 1816) jusqu'à des locaux supplémentaires pour que les troupes aient la possibilité d'étudier la science militaire lorsque le temps empêchait qu'elles puissent s'entraîner en plein champ (juillet-novembre 1816). Sans compter l'avance d'un supplément de mets pour le " thé dansant » donné par le lieutenant général von Gablenz (août 1817), les réquisitions de poêles, de chandelles, de lampes, que les habitants s'efforçaient de livrer en quantité suffisante pour satisfaire les occupants ${ }^{(11)}$.

Insatisfait du maire, qu'il jugeait insuffisamment diligent face à ses requêtes, le lieutenant Bucher gérait lui-même l'approvisionnement des troupes et les réparations et l'ameublement des bâtiments. En octobre 1816, ainsi, il se plaignait après avoir visité l'endroit choisi par le maire pour installer de nouvelles écuries: « Le charpentier et le maçon y travaillent avec une telle négligence et tout le bâtiment est aussi mal construit que je n'oserai jamais y loger les chevaux de Mr. le Colonel, que nous

8 Roger André, L’Occupation de la France par les Alliés en 1815 (juillet-novembre), Paris, É. de Boccard, 1924, p. 50.

9 Archives Municipales de Tourcoing (AMT), 5H/8: Cantonnement de troupes saxonnes: Avis du préfet du Nord (28 janvier 1816).

10 AMT, 5H/12 : Logement du Général en chef saxon cantonné à Tourcoing: Désignation et ameublement: Correspondance, délibérations municipales, liste d'objets, 1816-1818.

11 AMT, 5H/5 : Réclamations du lieutenant Bucher au maire de Tourcoing: Correspondance, 1816; 5H/19: Désignation d'un bâtiment pour servir de salle de théorie pour les soldats: Correspondance, 1816; 5H/27: Fournitures pour le thé dansant de la Mairie organisé par le lieutenant général: Correspondance, 7 août 1817 . 
attendons; je vous prierais de vouloir bien les engager pour qu'ils la construisent plus solidement et mettent en général plus d'application à leur ouvrage, sans quoi cette écurie ne pourra jamais servir ${ }^{(12)}$. Revendiquant la compétence pour assigner les logements de ses sous-officiers et soldats, il exigeait du maire qu'il donnât à ses adjoints et secrétaires les ordres les plus stricts de ne pas émettre de billet de logement en son nom (décembre 1817). Avec le maire, il intervenait lui-même pour s'occuper des affaires suivantes: l'enquête et les sanctions contre un bourgeois accusé d'avoir frappé deux soldats logés chez lui (octobre 1816); la surveillance médicale des prostituées, afin d'empêcher la propagation des maladies vénériennes parmi les troupes (décembre 1816 et été 1817); l'accrochage de lanternes sur les attelages pour éviter les accidents dans les rues sombres (décembre 1817); la surveillance des chiens de la commune, après qu'un de ses hommes eut été tué par un chien enragé (juin 1818) ${ }^{(13)}$. Même si des demandes similaires furent sans aucun doute faites, ici et là, par d'autres officiers, le ton de Bucher suggère que les Saxons prenaient un plaisir particulier à commander aux Français, compte tenu de leur relation tourmentée au proche passé. Les sources nationales le confirment également: dans un rapport sur Tourcoing établi en mars 1817, un commissaire régional de police regrette que, bien qu'il y ait au fond peu d'incidents sérieux entre les soldats saxons et les civils, "ils se montrent très exigeants sur toutes choses $»{ }^{(14)}$.

Néanmoins, les troupes saxonnes n’étaient pas sans commettre quelques " excès ». En avril 1817, un détachement saxon qui rentrait au pays, via l'arrondissement de Toul (Meurthe), commit contre les habitants des « désordres qui s'étaient à peine vus dans les moments les plus difficiles de la première invasion ». Selon un rapport du sous-préfet, " plusieurs de ces malheureux avaient le visage ensanglanté par suite des mauvais traitements qu'ils venaient d'essuyer; une femme fuyait précipitamment de sa maison pour se soustraire à la violence des soldats, qui exigeaient du vin, des liqueurs, du café " ${ }^{(15)}$. À Tourcoing même, à l'automne 1816, après un vif échange entre quelques Saxons éméchés et des soldats français, un Saxon tua d'un coup de sabre un dénommé Dutertre. Il fut puni d'une peine d'emprisonnement, en France jusqu'en 1818, puis en Saxe. De plus, selon les sources locales, il y avait des tensions sur le plan religieux entre des occupants luthériens et une population catholique ${ }^{(16)}$. Parfois, le comportement des troupes saxonnes était politiquement motivé, telle est du moins l'appréciation du commissaire général de police à Cambrai, ainsi qu'il le confie le 9 mars 1817 au ministre de la Police générale, Decazes : «Si on juge par les apparences, et le langage, les Saxons sont faiblement portés pour le gouvernement. Ils affectent souvent de regretter un homme [Napoléon] qui, sans doute, se rappelle Leypsick [Leipzig] et ne les paye pas d'un regret

12 AMT, 5H/5 : Réclamations du lieutenant Bucher au maire de Tourcoing : Correspondance, 1816.

13 Lettre du commissaire spécial du service extraordinaire au Ministre de la Police, Lille, 16 mars 1817, Archives Nationales de France [A.N.], F7/9901 (Nord).

14 A.N., F7/9899 (Meurthe).

15 A.N., F7/9899 (Meurthe).

16 Olivier Podevins, «L'occupation de Tourcoing par les troupes saxonnes entre 1816 et 1818: Un épisode peu connu de l'histoire de la ville », article réalisé au sein du groupe de recherche sur les relations France-Saxe, passées et actuelles, à l'Université technique de Dresde (Saxe), et trouvé aux Archives municipales de Tourcoing. Voir notamment p. 16-17. 
semblable " ${ }^{(17)}$. «Vainqueur défait », ainsi que le nomme le spécialiste d'histoire locale Olivier Podevins, le Saxon était occupant à contrecœur. Moins brutal que le Prussien, il n'en montra pas moins un penchant revanchiste entre 1815 et $1818^{(18)}$.

\section{La Bavière}

Pour des raisons semblables, le contingent bavarois était même plus belligérant vis-à-vis des Français, en dépit du fait que les deux pays étaient alliés depuis le traité de Bâle en 1796. Le royaume avait prospéré sous la férule du prince Max Joseph, monté sur le trône en 1799. Il s'était agrandi au moment du recès d'Empire (Reichsdeputationshauptschlu $\beta$ ) de 1803, devenant le troisième derrière la Prusse et l'Autriche pour la superficie de son territoire au sein du Saint-Empire, le premier au sein du Rheinbund où Max Joseph avait pris le titre de roi en 1806. Le royaume devint constitutionnel en 1808, à l'inspiration mais non à l'instigation de la France, et le seul de ce type dans cette partie de l'Europe ${ }^{(19)}$. Allié indéfectible de l'Empire, la Bavière prit sa part de la malencontreuse campagne de Russie. Mais au lendemain de la débâcle, elle fut l'un des premiers États de la Tierce Allemagne à faire défection, signant un traité avec la Prusse, l'Autriche et la Russie le 8 octobre 1813 et huit jours avant que les Alliés n'engagent la bataille des nations. Malgré les lourdes pertes de 1812-1813, l'armée bavaroise mit cependant sur le terrain 40000 hommes (dont la moitié venait d'une nouvelle Légion mobile, commandée par le général Karl von Wrede) pendant l'invasion de l'hiver 1814. Après les Cent-Jours, pendant l'été 1815, elle envoya encore en France 60000 hommes ${ }^{(20)}$. Selon les dispositions de l'occupation de garantie, où elle ne prit part qu'avec certaines réticences, la Bavière gardait sur place 10000 hommes, soit tout de même deux fois plus que les puissances de rang secondaire ${ }^{(21)}$.

Avec de tels effectifs, il n'est pas étonnant que les troupes bavaroises aient causé beaucoup de dégâts en 1814 et surtout en 1815, quand elles furent lancées sur une large bande de territoire depuis la Seine-et-Marne, à l'est, entre les forces prussiennes et les forces autrichiennes. Les Bavarois créèrent un commandement général, sous la férule de von Wrede, qui resta fameux pour avoir saisi le produit des impôts dans sa zone, menaçant les receveurs et les fonctionnaires locaux qui se mettaient sur son chemin de l'« exécution militaire ». À Meaux, un général bavarois frappa les habitants d'une contribution exorbitante de $2 \mathrm{M}$ de francs, et lorsque le sous-préfet refusa de la collecter, il fut emprisonné à Melun, avant d'être finalement libéré sur intervention

17 A.N., F7/9901 (Nord).

18 O. Podevins, «L'occupation de Tourcoing par les troupes saxonnes » (note 16), p. 13.

19 J. Sheehan, German History, 1770-1866 (note 5), p. 261-262.

20 Michael V. Leggiere, The Fall of Napoleon: The Allied Invasion of France, 1813-1814, Cambridge, Cambridge University Press, 2007, p. 125, et R. André, L'Occupation (note 8), p. 50.

21 Voir ainsi la lettre de Wellington au maréchal prince Wrede, 5 novembre 1815, in: WeLLINGTON, Dispatches, Londres, John Murray, 1837, vol. 12. Sur les troupes bavaroises d'occupation, voir également Oskar Bezzel (dir.), Geschichte des Bayerischen Heeres unter König Max I. Joseph von 1806 bis 1825, vol. 6, t. 1, Munich, Bayerischen Kriegsarchiv, 1933, et Emmanuel Boudot, «L'occupation alliée de 1815-1818 dans le département de la Moselle ", mémoire de maîtrise d'histoire sous la direction d’Alfred Wahl (Université de Metz, 1995), Archives départementales de la Moselle. 
du colonel de la gendarmerie bavaroise, le major-général Reckberg ${ }^{(22)}$. Début août, en Bourgogne, plusieurs petites villes situées entre Dijon et Semur-en-Auxois furent " écrasées » par les demandes bavaroises, qui les mettaient en demeure de loger pas moins de deux ou trois régiments entiers. Selon le rapport d'un conducteur de diligence témoin des faits: "Semur est également encombré; les soldats se livrent à toutes les horreurs possibles, ils pillent et violent partout " ${ }^{(23)}$. À la même époque, à Barsur-Aube, un voyageur rapporta qu'un gros village, à peu de distance de la cité, avait été brûlé par les Bavarois et que les habitants qui avaient tenté de résister avaient été massacrés par un détachement spécialement envoyé par l'officier commandant. Selon le même témoin, un village avoisinant nommé Dieuville avait également été incendié, comme il l'avait été déjà en 1814 (ce qui laisse supposer que la mémoire de ces faits informait la conduite des troupes, comme celle des habitants $\left.{ }^{(24)}\right)$. À une date aussi tardive que le 22 décembre 1815, les habitants de la Meurthe se plaignaient de ce que leur département, pourtant en principe exempt de l'occupation de garantie, le long de la frontière, était encore occupé par 10000 Bavarois, qui ne se préparaient nullement à rentrer chez eux, ajoutés à des passages de milliers d'hommes des troupes saxonnes et russes qui causaient « tous les désordres inévitables; des dégâts dans les forêts, des vols sur les routes ${ }^{(25)}$. Les habitants de Lunéville, à la toute fin de l'année, étaient si impatients de voir partir la garnison bavaroise qu'ils pétitionnèrent à cette fin à la fois auprès du roi de France et du roi de Bavière ${ }^{(26)}$.

Les violences des Bavarois ne devaient pas cesser avec la transition vers l'occupation régulière, au début de 1816, lorsqu'ils furent limités à 10000 hommes et cantonnés dans la région de Sarreguemines. Au cours des trois années d'occupation, de nombreux abus leur furent attribués, comme aux Russes et aux autres Allemands présents dans la région. Début 1817, selon des extraits de lettres de la région de Strasbourg envoyées au ministère de la Guerre à Munich, les habitants de Sarreguemines se plaignaient des «vexations » qu'ils avaient endurées sans discontinuer au cours des 18 mois écoulés; « dernièrement encore, disait un de ces textes, des officiers à demi-solde, insultés par des officiers bavarois, leur ont proposé la partie d'honneur; mais ceux-cy ont apposté [sic] des hommes armés, qui, lorsqu'ils rentraient paisiblement chez eux à 10 heures du soir, les ont accablés de coups; s'ils ne sont pas morts sous les coups, ils sont au moins bien malades! Je sais que ce n'est pas là l'intention de votre bon et excellent Roi, mais il existe 20 Plaintes de ce genre, et on n'a encore fait droit à aucune! »(27) En mars de la même

22 Analyse de la correspondance au ministère de la Police, Seine-et-Marne, 13 et 15 juillet 1815, A.N., F7/3147. Sur les saisies des revenus publics, voir aussi Bayerisches Hauptstaatsarchiv Kriegsarchiv, Alter Bestand B, liasse 592: Krieg geg. Frankreich, 1815, XIII. Generalgouvernement Auxerre: Administration d. occupierten Franz. Departements I. Abteilung.

23 A.N., F7/3824, Dijon, départ du $1^{\mathrm{er}}$ août 1815.

24 A.N., F7/3824, Bar-sur-Aube, arrivée du 6 août 1815.

25 Rapport hebdomadaire du département de la Meurthe, 22 décembre 1815, A.N., F7/3823.

26 A.N., F7/3825, Strasbourg, départ du 21 décembre 1815.

27 Bayerisches Hauptstaatsarchiv Kriegsarchiv, Alter Bestand B, liasse 599: Krieg geg. Frankreich, 1813/1815: Occupationstruppen, 1815-1818. Les débordements des Bavarois sont également documentés dans les Archives municipales de la ville de Sarreguemines : Section II : 2D1-9 et Section III : 4D1-10 (Correspondance du Maire), ainsi que Section II : 9H35-80 (Événements militaires). 
année, le ministère de la Police générale reçut de nombreuses plaintes au sujet du commandement bavarois à Remering, qui défiait les autorités locales en faisant arrêter le sergent de police lorsque celui-ci affichait les annonces de la mairie ${ }^{(28)}$. Aussi tard qu'en été 1818 , alors même que les troupes s'apprêtaient pour l'évacuation définitive de la France, nombre d'excès furent encore à déplorer de la part de soldats bavarois, parfois ivres, parmi lesquels: l'attaque d'un jeune homme à coups de couteau; la tentative de viol d'une femme qui rentrait des champs; les provocations à l'égard des filles lors des danses dans les cabarets; l'attaque du maire de Roppewiller sur la route; et les brimades à l'égard de deux conducteurs de voitures, dont les soldats voulaient couper les traits des chevaux. Cette dernière affaire, jugée par une cour martiale, aboutit à la condamnation de trois hommes à plusieurs jours de fers, et à celle de leur officier à des dommages et intérêts ${ }^{(29)}$.

Tout le long de l'occupation, l'un des abus le plus souvent déplorés de la part des Bavarois était la propagation de rumeurs relatives au retour de Napoléon. Que ces anciens alliés du Grand Empire l'aient regretté réellement, ou qu'ils aient surtout voulu provoquer les Français, quoi qu'il en soit il circulait de très nombreuses anecdotes à son sujet, laissant imaginer qu'il s'était échappé de l'île d'Elbe et qu'il en résulterait une nouvelle guerre contre la France. À l'automne 1815, près de Paris, un contingent bavarois avait montré de l'enthousiasme pour l'Usurpateur ${ }^{(30)}$. En décembre, dans la Meurthe, un fonctionnaire de police locale rapportait que « les Bavarois qui occupent ce département y tiennent les propos les plus indécents contre la famille Royale, et se montrent partisans zélés de l'usurpateur. Ils répandent que Bonaparte n'est point à Sainte Hélène; qu'il sera à Paris au mois de mars; que les Saxons, les Wurtembergeois, les Polonais se joindront à son armée, etc. Ces propos absurdes sont communs à tous les soldats de cette nation ${ }^{\left({ }^{(1)}\right.}$. Le même genre de bruits de guerre étaient encore diffusés par des Bavarois en juin 1816 à Strasbourg: "On dit aujourd'hui, à ce sujet que le roi de Bavière, le prince Charles [de Habsbourg] et le prince Eugène [de Beauharnais] sont les protecteurs du fils de Bonaparte; qu'ils se sont juré par un pacte signé de le rétablir sur le trône de France et qu'ils travaillent de concert pour réaliser ce grand projet " ${ }^{(32)}$. Et en février 1818 encore, dans le Haut-Rhin, « le bruit est répandu à Mulhausen que le roi de Bavière fait de grands préparatifs de guerre; et que c'est le prince Eugène qui doit avoir le Commandement de l'armée qu'il met sur pied " ${ }^{(33)}$. Comme ces fausses nouvelles troublaient beaucoup les habitants, les autorités faisaient enregistrer des plaintes auprès des commandants alliés. Bref, à travers ces provocations physiques et verbales, les forces bavaroises d'occupation travaillaient à humilier leurs anciens alliés.

\footnotetext{
28 A.N., F7/9900 (Moselle).

29 A.N., F7/9900 et 9904.

30 A.N., F7/3823, Seine-et-Marne, 9 novembre 1815.

31 A.N., F7/3823, Meurthe, départ du 14 décembre 1815.

32 A.N., F7/3826, Strasbourg, départ du 2 (juin 1816).

33 A.N., F7/3827, Mulhouse, départ du 13 (février 1818).
} 


\section{Le Wurtemberg}

Mais les pires excès furent sans doute commis par les troupes wurtembergeoises, qui occupaient le nord-est de l'Alsace, juste du côté opposé de la frontière de leur patrie. Ce petit État avait tiré avantage de la dislocation du Saint-Empire pour mettre en route une politique d'expansion et de centralisation. Arrivé au pouvoir en 1797, le duc Frédéric II avait été couronné roi en 1806, en reconnaissance d'avoir rejoint l'alliance anti-autrichienne. Il fournirait de nouvelles troupes au Grand Empire en 1807 contre la Prusse, en 1809 de nouveau contre l'Autriche, en 1812 contre la Russie ${ }^{(34)}$. Le Wurtemberg passa dans le camp allié juste à temps, alors que les puissances préparaient l'invasion de la France à la fin de 1813, de sorte que Frédéric chercha à montrer du zèle pour gagner la faveur du tsar. Dans le cadre de l'invasion, ses troupes étaient rattachées à l'Armée de Silésie commandée par Schwarzenberg, qui traversa le cours haut du Rhin pour entrer en Alsace et en Franche-Comté. Il fournit le double du contingent qui lui avait été fixé: 12250 hommes pour l'infanterie de ligne, 12250 membres de la milice, et 24 canons $^{(35)}$. À la fin de 1815, le Wurtemberg apporta 20000 hommes à la deuxième invasion de la France ${ }^{(36)}$. Sa part dans l'occupation de garantie fut fixée à 5000 soldats, stationnés dans la région de Wissembourg.

Dès le début de la deuxième occupation, ces hommes furent fameux pour leurs exigences et pour leur dureté. Dans les premiers temps de l'invasion, ils avaient harcelé les autorités locales de demandes et de réquisitions. À Issenheim, selon une plainte reçue par le préfet du Bas-Rhin, le maire ayant refusé de satisfaire à la longue liste de demandes qu'on lui avait soumises (jusqu'à des réparations à faire sur les schakos), sans un ordre écrit du commandement allié, leur officier commandant installa chez lui 18 soldats, qui allèrent se servir dans ses réserves et dans sa cave jusqu'à ce qu'il obtempère $^{(37)}$. Les Wurtembergeois terrorisaient aussi la population locale. Près de Châlons-sur-Saône, en août 1815, un conducteur de diligence les avait déjà signalés, par opposition aux troupes autrichiennes, " assez modérés »: "Les Wurtembergeois continuent leurs excès à Autun; ils accablent de coups de bâtons les particuliers; on cite entre autres un homme de 75 ans, qui est mort, à la suite de ceux dont il a été frappé. À Saulieu, une femme est morte, et deux autres ont été laissées sans connaissance, à la suite des brutalités de ces mêmes soldats. Ils violent partout " ${ }^{(38)}$. Un maire redoutait même que ces abus ne s'aggravent encore après le départ des Autrichiens, au cours de la transition qui devait conduire à l'occupation de garantie ${ }^{(39)}$. En fait, les

34 J. Sheehan, German History (note 5), p. 263-264. Sur le rôle du Wurtemberg dans l'occupation du BasRhin, voir aussi Gustave Groeber, "L'Occupation d'Oberbronn par des troupes wurtembergeoises de 1816 à 1818 », Bulletin de la Société Niederbronnoise d'histoire et d'archéologie, 8 (1960), p. 182207, et Daniel Peter, "Les occupations alliées dans l'arrondissement de Wissembourg, 1814-1818 », L'Outre-Forêt. Revue d'histoire de l'Alsace du Nord, 58 (1987), p. 48-52.

M.V. Leggiere, The Fall of Napoleon (note 20), p. 125 sq.

R. ANDRÉ, L'Occupation (note 8), p. 50.

Archives départementales du Haut-Rhin (Colmar) (ADHR), 8 R 33, lettre du maire d'Issenheim au préfet, 16 septembre 1815 .

A.N., F7/3824, Châlon-sur-Saône, arrivée du 4 août 1815.

ADHR, 8R/33, lettre du maire de Kirrwiller au baron de Greiffenegg, commandant du gouvernement de Colmar, 14 septembre 1815. 
troupes du Wurtemberg commirent encore de nombreux « excès » au moment de leur évacuation, à la fin de 1815. Selon l'historien alsacien Bernard Vogler, à ce momentlà, la zone traversée par les forces commandées par le prince Frédéric lui-même fut particulièrement mal traitée ${ }^{(40)}$.

Dans ces environs de Wissembourg, les rapports entre les habitants et les troupes d'occupation allemandes furent parmi les pires de tous les secteurs répartis entre les Alliés. Jusqu'en 1818, il y eut sans cesse des conflits, dégénérant souvent en rixes, autour des réquisitions de nourriture, de matériel et de moyens de transport, mais aussi autour des dommages causés aux biens, des règles douanières, des impôts sur les boissons, des droits d'usage forestiers, et enfin autour de la religion et des femmes, sans parler des insultes verbales - bien réelles ou perçues comme telles. Au printemps 1816, près de la ville de Niederbronn, il y eut de nombreux affrontements entre les douaniers français et les soldats wurtembergeois, qui passaient des marchandises en contrebande, avec la complicité souvent de quelques habitants, principalement des femmes, plus familières avec ces interlocuteurs germanophones quavec les fonctionnaires bourboniens qui cherchaient à remettre en route la perception des impôts. Ces tensions firent au moins un mort, fin avril $1816^{(41)}$. En janvier 1818, dans la commune voisine de Niederroeden, une vingtaine de soldats wurtembergeois quittèrent leur cantonnement de Seltz et marchèrent sur le village, entraînant derrière eux une centaine d'autres, stationnés aux alentours. Ils encerclèrent l'église et firent tant de bruit que les fidèles durent venir dehors les affronter. Ils les attaquèrent alors à coup d'épée, provoquant une mêlée où l'on releva au moins une femme et six hommes blessés, dont trois vieillards. Le maire se plaignit au sous-préfet: "Je ne saurais vous exprimer la terreur que cette action horrible a produit; comme au milieu de la guerre la Consternation était générale » ${ }^{(42)}$. De culture allemande commune mais le plus souvent d'affiliation religieuse différente, Wurtembergeois et Alsaciens se montrèrent donc particulièrement méfiants et violents les uns envers les autres.

Le temps ne fit pas baisser le nombre d'incidents. À mesure que le terme de leur présence approchait et qu'ils devenaient plus inquiets de leur propre avenir, en tant qu'armée et en tant qu'État, les Wurtembergeois semblaient de plus en plus provocateurs envers leurs hôtes français. À Soultz, à l'automne 1818, certains rentraient d'une

40 « Le retour des troupes alliées provoque à nouveau une multiplication de réquisitions dans les campagnes, en particulier dans l'arrondissement de Wissembourg qui doit fournir un millier de manœuvres par jour et 50000 francs par mois. " (Bernard VoGLer, Histoire politique de l'Alsace, de la Révolution à nos jours. Un panorama des passions alsaciennes, Strasbourg, La Nuée Bleue, 1995, ch. 3).

41 Sur ces tensions entre les Wurtembergeois et les autorités locales, voir ADBR, RP 1247, ainsi que les Archives municipales de Wissembourg, et le Hauptstaatsarchiv Stuttgart, notamment E 289 a, liasse 277: Bezeichnung des Bestands, Mobile Kommandobehörden I: Conceptbuch der allgemeinen Correspondenz mit verschiedenen Behörden außer dem Corp, Juni 1816-April 1817; E 289 a, liasse 283: Bezeichnung des Bestands, Mobile Kommandobehörden I: Korrespondenz mit der Präfektur Straßburg, Nov. 1815-Nov. 1818; et E 289 a, liasse 284: Bezeichnung des Bestands, Mobile Kommandobehörden I: Correspondenz mit dem Unterpräfektur Weißenburg, Dez. 1815-1818.

42 Rapport du 25 janvier 1818, Hauptstaatsarchiv Stuttgart, E 270 a, liasse 466 : Geheime Kriegskanzlei. Sur cet incident, cf. également Hauptstaatsarchiv Stuttgart, E 270 a, liasse 472 : Geheime Kriegskanzlei, Occupationkorps im Elsaß : Korrespondenz mit dem Hauptquartier des Felmarschalls Herzog v. Wellington während der Okkupation Frankreichs durch die Allierten, 1816-1818. 
revue passée par Wellington lorsqu'ils commirent quelques « excès » dans une auberge où se célébrait un mariage juif. Après en avoir forcé l'entrée,

« Ils ont mis le désordre dans l'assemblée en forçant les femmes à danser et le maître de l'hôtel, étant monté pour rétablir la tranquillité, a reçu un coup de sabre sur le nez. Les voisins éveillés par le bruit sont accourus et ont frappé à leur tour les soldats wurtembergeois dont six ont été grièvement blessés. Il paraît que les habitants de ce canton attendent avec une impatience extrême le moment où ils seront débarrassés de l'armée d'occupation » ${ }^{(43)}$.

Autour des mêmes dates, une rixe éclatait à Froeschwiller, pour laquelle le préfet du Bas-Rhin établit un rapport:

"Le jour de la fête de cette commune, deux soldats se présentèrent à l'entrée de la salle de danse. Le garçon qui faisait les honneurs de cette salle, suivant l'usage du pays, les invita, avec une extrême politesse, à passer dans la salle voisine, afin d'éviter toute dispute, mais ils prirent cette invitation en mauvaise part et, loin d'y déférer, quoique ceux de leurs camarades qui étaient restés dans la rue les y invitassent, ils répondirent par des injures et entreprirent d'entrer par la force. Dès lors, il s'y engagea une rixe à la suite de laquelle trois jeunes gens et plusieurs soldats furent blessés. Le lendemain, vinrent dans le village une soixantaine de soldats qui entrèrent dans la cour de la maison dans laquelle les jeunes gens étaient rassemblés. L’entrée ne put leur être refusée, parce que cette maison est publique. Une femme étant entrée pour retirer ses deux fils qui se trouvaient dans cette maison, fut frappée de manière à être renversée. Les jeunes gens qui se trouvaient en nombre inférieur quittèrent alors la salle et furent poursuivis dans les rues par les soldats. Quatre personnes, dont une femme et un vieillard, furent blessés » ${ }^{(44)}$.

Et de tels désordres étaient encore rapportés à Reichshoffen ou à Wissembourg, où des jeunes gens s'étaient rassemblés pour la loterie de l'armée, en octobre 1818, à quelques journées seulement de l'évacuation du territoire français par les troupes étrangères ${ }^{(45)}$. Ainsi que l'histoire locale, en la personne de Gustave Groeber, l'a rapporté, de tels incidents provoqués par d'anciens alliés, dont un certain nombre portaient la légion d'honneur, étaient vus d'un particulièrement mauvais œil par les populations locales, d'autant que celles-ci comptaient dans leurs rangs d'anciens soldats de la Grande Armée ${ }^{(46)}$.

\section{Conclusion}

Comme l'illustrent ces forcément brefs extraits, le monopole de la violence n'était nullement du côté des Prussiens (ou des Autrichiens) dans le cadre de l'occupation de la France impériale vaincue. Il faut noter, sans doute, que les troupes bavaroises, wurtembergeoises ou saxonnes donnèrent parfois des preuves de compassion et furent capables de prêter assistance aux habitants, offrant leurs bras pour lutter contre les incendies ou pour rentrer les moissons, ou apportant des secours individuels à des malades ou des indigents. De même, les troupes issues de la Tierce Allemagne apparaissent ni plus ni moins brutales que celles des grandes puissances occupantes. Dans

\footnotetext{
43 A.N., F7/9904 (Bas-Rhin).

44 Ibid.

45 Ibid.

46 G. Groeber, « L'occupation d'Oberbronn » (note 34), p. 194.
} 
leur comportement, ces troupes étaient bien marquées par leurs expériences pendant les dernières années des guerres napoléoniennes. Alliées à la France entre 1806 et 1812, parfois sans enthousiasme, ces troupes avaient connu un revirement d'allégeance brusqué, en l'espace de quelques semaines voire de quelques jours, en 1813, pour passer du côté de la coalition. Cela fut décisif pour expliquer leur attitude de vainqueurs, en 1814. De même, l'expérience et la mémoire de 1814 ont formé les occupants de 18151818 , qu'ils eussent ou non directement pris part à la première invasion. En 1815, il y a de nombreuses preuves que la rudesse du comportement des troupes fut proportionnée au degré de résistance que les régions envahies avaient offertes en 1814. Bien qu’il $\mathrm{y}$ ait encore des recherches à faire sur les mentalités des divers occupants, il est certain que la rupture de 1813-1814 a bien conditionné le comportement des troupes en France pendant l'occupation de 1815-1818.

Traduit de l'américain par Nicolas Bourguinat

\begin{abstract}
During the collapse of the Empire in 1814 and again in 1815, the Prussian and, to a lesser extent, the Austrian invaders are known to have been particularly violent toward the French. Less well studied is the role of forces from the "Third Germany" in the occupations of France in 1814 and 1815-1818. With the help of local and national police and judicial archives as well as German military records, this article examines the cases of three smaller powers involved in these occupations: Saxony, Bavaria and Württemberg. Describing the relations between occupiers and occupied in their zones, it argues that these former allies were no more friendly toward the French than their long-time enemies.

\section{Résumé}

Pendant la déprise de l'Empire en 1814 et encore en 1815-1818, les envahisseurs prussiens et, dans une moindre mesure, autrichiens sont connus pour avoir été particulièrement violents envers les Français. Moins bien étudiées sont les forces de la "Tierce Allemagne " dans les occupations de France en 1814 et 1815-1818. Employant des archives policières et judiciaires locales et nationales, ainsi que des documents militaires allemands, cet article analyse les cas de trois petites puissances qui ont participé à ces occupations : la Saxe, la Bavière et le Wurtemberg. Décrivant les rapports entre occupants et occupés dans leurs zones, il montre que ces anciens alliés étaient aussi durs avec les Français que leurs ennemis traditionnels.
\end{abstract}

